\title{
RAPID VARIATIONS IN INTERGRANULAR SPACE
}

\section{A. NESIS}

Kiepenheuer Institute for Solar Physics, W-7800 Freiburg, Germany

A. HANSLMEIER

Institute for Astronomy, A-8010 Graz, Austria

R. HAMMER

Kiepenheuer Institute for Solar Physics, W-7800 Freiburg, Germany

R. KOMM

National Optical Astronomy Observatories, Tucson, AZ 85719

W. MATTIG AND J. STAIGER

Kiepenheuer Institute for Solar Physics, W-7800 Freiburg, Germany

ABSTRACT Spectrograms of high spatial resolution taken every $15 \mathrm{~s}$ reveal rapid variations of the continuum intensity and line width. Variations of the latter seem to be related to Doppler velocity gradients.

\section{INTRODUCTION}

Excellent spectrograms taken with the VTT in Izaña (Tenerife) enable us to study in detail the dynamics of intergranular space, especially the transition from intergranular space to the granule. So far, our investigation of these spectrograms concerned the phenomenology of the granulation (cf. Nesis et al. 1992a) and its dynamics (cf. Nesis et al. 1992b). In this investigation we are interested in the temporal aspects of intergranular space. We use a series of five spectrograms which cover a time interval of $1 \mathrm{~min}$, taken at the same position of the solar disk every $15 \mathrm{~s}$.

Our primary intention was to look for rapid variations within the intergranular space. We address the following questions:

(i) Are there short-term variations (of the order of $1 \mathrm{~min}$ ) of the intensity, velocity, or the full width at half maximum (fwhm)?

(ii) Are there positions in the photospheric layers where rapid variations of these quantities are preferentially located?

(iii) Are there physical processes which can be associated with rapid time changes of these quantities? 

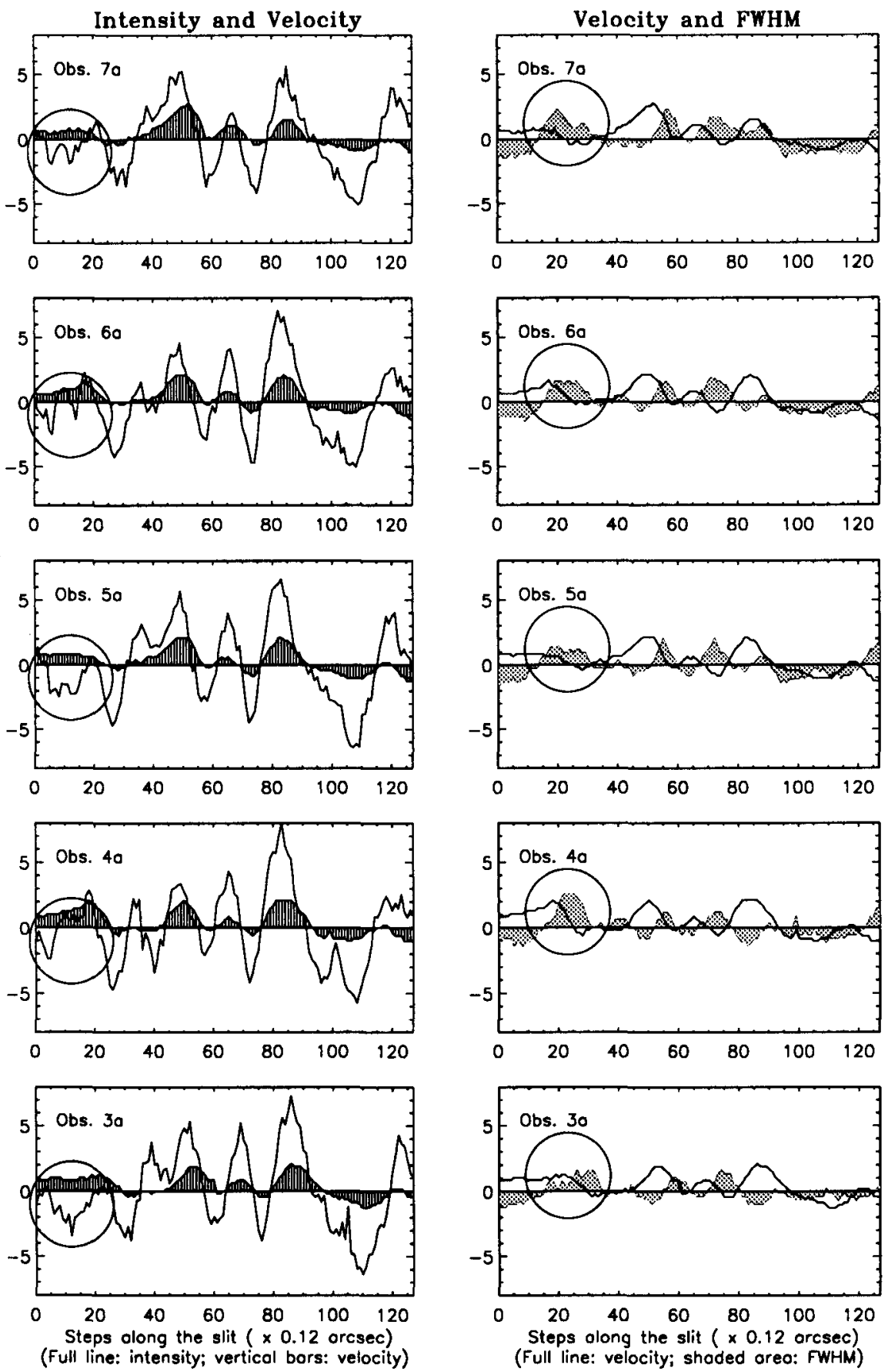


\section{RESULTS AND DISCUSSION}

The data processing in this investigation is straightforward and includes the calculation of the continuum intensity, Doppler velocity and full width at half maximum (fwhm) of the absorption line $\lambda 4912.03 \AA(\mathrm{g}=0)$, along the slit of the spectrograph. The two columns of Fig. 1 show the intensity and velocity (left column) and the velocity and fwhm (right column) for the five successive spectrograms (Obs 3 - Obs 7) for a part of the entire slit; the findings, however, are representative for the entire slit. Referring to the questions in the introduction, our results can be summarized as follows:

(i) Along the slit we find cases (which we have denoted by circles) where the intensity drastically changes its value within $60 \mathrm{~s}$. Furthermore, we find variations of the line width fwhm (again denoted by circles) which are obviously associated with variations of the velocity gradient. It is noteworthy that the drastic changes of intensity and line width are not accompanied by comparably rapid changes of the Doppler velocity.

(ii) The rapid intensity variations (circles, left column) are located in the intergranular space. Rapid variations of fwhm (circles, right column) take place in the transition zone between granule and intergranular space, i.e. where the velocity changes its sign, and where large velocity gradients exist.

(iii) The rapid variations of both intensity and fwhm reflect the importance of the intergranular space, especially the transition from granule to intergranular space. As for physical processes which could be associated with rapid changes in time, we suggest a causal relation between intensity, fwhm, and velocity gradients. Such a relationship could be the result of shear instability, or of an emergence of new small granules in an older granular velocity environment. Horizontal "intensity" waves traveling with sound speed across the spectrograph slit might also be relevant, as suggested by Title (1992).

A theoretical explanation of these findings involves the interplay between thermodynamical and hydrodynamical processes in intergranular space. The fact that the velocity gradient varies suggests the build-up of shear layers at the transition from intergranular space to granule, which enhances the local turbulence. On the other hand the rapid intensity variations could be an indication of short-living granules, or intensity features traveling across the slit with sound speed.

\section{REFERENCES}

Nesis, A. Hanslmeier, A., Hammer, R., Komm, R., Mattig, W, and Staiger, J. 1992a, A\&A, 253, 561

Nesis, A. Hanslmeier, A., Hammer, R., Komm, R., Mattig, W, and Staiger, J. $1992 \mathrm{~b}, \mathrm{~A} \& \mathrm{~A}$, to be published

Title, A. 1992, private communication 